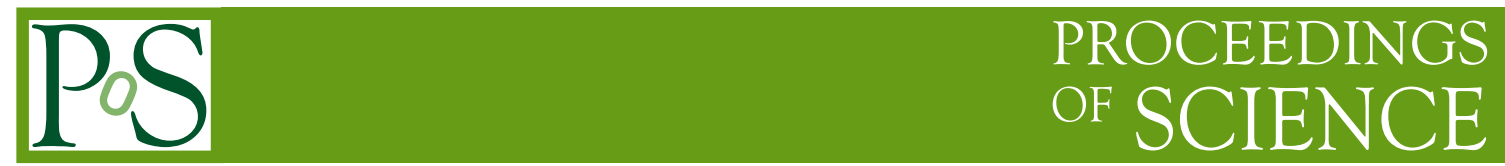

\title{
Measurements of Neutrinos at the Angra Reactor
}

\author{
Pietro Chimenti*广 M.A.Leigui de Oliveira, R.M.Lima for the Angra Collaboration \\ Universidade Federal do ABC-Brazil \\ E-mail: pietro.chimenti@ufabc.edu.br
}

The Angra Project aims at measuring the anti-neutrinos produced by the nuclear power plant situated near the Angra dos Reis city. In the present article we discuss some recent developments on the simulation of the detector being developed as part of the design study.

5th International School on Field Theory and Gravitation

April 20-24, 2009

Cuiabá city, Brazil

\footnotetext{
*Poster session.

${ }^{\dagger}$ The author would like to thank the Brazilian agencies FAPESP, CAPES and CNPq.
} 


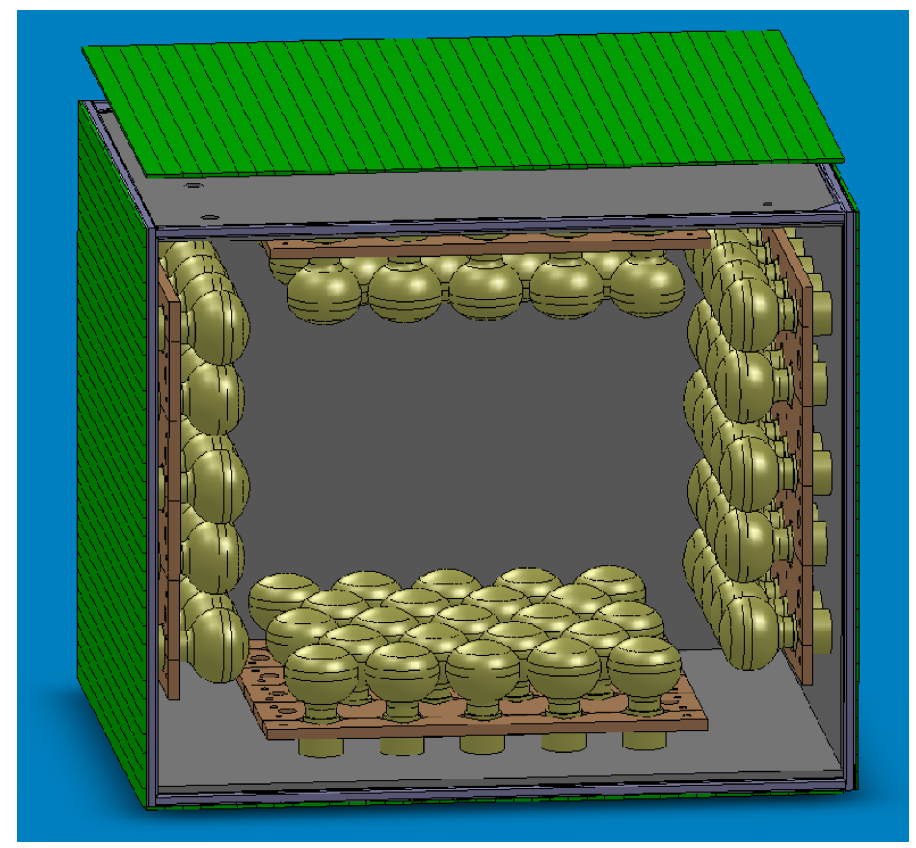

Figure 1: Design of the Very Near Detector (the external shield is not included in this drawing).

\section{Introduction}

Nuclear reactors played an important role in neutrino physics, both in the discovery and in the

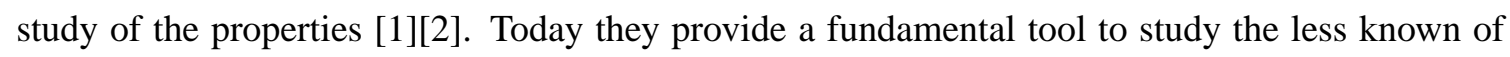
the mixing angles: $\Theta_{13}[$ [3] [ $[$ ]. Conversely (Anti-)Neutrinos carry information which have been proved useful for the understanding of the activity of reactors cores in such a way that important applications can today be developed in the field of nuclear nonproliferation [5]. In this contribution we describe the recent developments in the simulation of the Angra Experiment [6][7][8][9], a project to observe anti-neutrinos at the Angra dos Reis nuclear complex in Brazil.

In the long term it is planned to place in Angra three detectors at three baselines: the Very Near Detector (VND) at a distance from the core of 30-40m, the Near Detector (ND) at a distance from the core of about $300 \mathrm{~m}$ and the Far Detector (FD), under the hill "Morro do Frade" and at a distance from the core of $1.0-1.5 \mathrm{Km}$. The three detectors have different purposes: the VND, with a mass of 1Tonn approximately, will serve as a prototype for the other two bigger ones and also as a development for nonproliferation techniques. Both the ND and FD will provide a precision measurement for oscillation studies. As for security reason it will not be possible to place any low flash-point material (i.e. liquid scintillator) at a short distance from the nuclear reactor core, the possibility to build a VND based on the water Cerenkov technique is under study with both Monte-Carlo simulations and small prototypes development.

\section{The simulation}

The proposed design of the VND (fig.11) is an assembly of three subsystems: the central detec- 


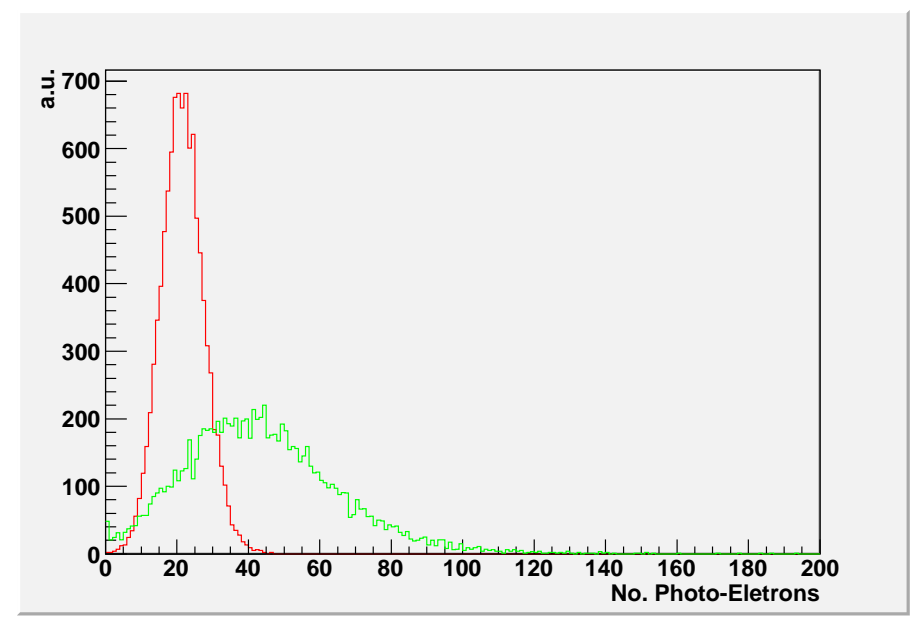

Figure 2: Distributions of photo-electrons due to positrons (in red) and neutrons (in green) produced in the fiducial volume.

tor (based on the water Cerenkov technique), the veto (based on extruded scintillator detectors) and an external passive shield to protect the inner parts from the low energy external natural radioactivity.

The central detector, a tank about $1.80 \mathrm{~m}$ long $1.4 \mathrm{~m}$ wide and $1.4 \mathrm{~m}$ height, contains 74 photomultipliers (PMTs) Hamamatsu R5912 disposed on four sides of the tank (as visible in fig.11). The dimensions of the tank are such that an internal fiducial volume of $1 \mathrm{~m}^{3}$ can be defined in such a way to have about $10^{3}$ neutrino events per day (considering the distance at the very near site and the $4 G W_{t h}$ of reactor power). Moreover it is filled with a mixture of water with a small percentage of Gadolinium in order to enhance the neutron signal and exploit the timing correlation between the prompt signal due to positrons and the delayed signal due to neutrons.

The simulation described in this contribution makes uses of the Geant4[10] package in order to predict the expected distribution of photo-electrons in the central detector due to positrons of $2 \mathrm{MeV}$ energy (this is the characteristic energy of the positrons produced in the inverse beta decay) and thermal neutrons. Developing the code, particular attention has been paid to: the list of included processes which is the most complete commonly used generic list (naturally including the optical processes); the accurate description of the geometric set-up; the proper description of the optical characteristics of the materials (including the quantum efficiency of the PMTs).

The results are shown in fig.2. In red is visible the distribution of photo-electrons (p.e.) due to positrons produced inside the fiducial volume. On average it is expected a signal of about 20 p.e.. In green is the distribution due to thermal neutrons. Although in this case the mean signal is higher (about 40 p.e.) the distribution is broader also due to fluctuations in the number of gammas emitted by the Gd.

\section{Conclusions}

The Angra Project is in the phase of design of the first detector to be placed at the very near site. Simulations are on going to estimate the signal to noise ratio of this detector. The next steps 
toward this goal are the estimate of the noise due to natural radioactivity.

\section{References}

[1] T. Kajita, "Discovery of neutrino oscillations," Rept. Prog. Phys. 69 (2006) 1607.

[2] U. Dore and D. Orestano, "Experimental results on neutrino oscillations," Rept. Prog. Phys. 71 (2008) 106201 [arXiv:0811.1194 [hep-ex]].

[3] F. Ardellier et al. [Double Chooz Collaboration], "Double Chooz: A search for the neutrino mixing angle theta(13)," arXiv:hep-ex/0606025.

[4] X. Guo et al. [Daya-Bay Collaboration], "A precision measurement of the neutrino mixing angle theta(13) using reactor antineutrinos at Daya Bay," arXiv:hep-ex/0701029.

[5] A. Bernstein et al., "Nuclear Security Applications of Antineutrino Detectors: Current Capabilities and Future Prospects," arXiv:0908.4338 [nucl-ex].

[6] E. Casimiro and J. C. Anjos [Angra Collaboration], "The Angra Neutrino Project: Precise Measurement Of Theta(13) And Safeguards Applications Of Neutrino Detectors," AIP Conf. Proc. 1116 (2009) 251.

[7] E. Casimiro and J. C. Anjos, "Cosmic muon background and reactor neutrino detectors: The Angra experiment,” J. Phys. Conf. Ser. 116 (2008) 012003.

[8] J. C. Anjos et al., "Angra dos Reis reactor neutrino oscillation experiment," Braz. J. Phys. 36 (2006) 1118.

[9] J. C. Anjos et al., "Angra neutrino project: Status and plans," Nucl. Phys. Proc. Suppl. 155 (2006) 231 [arXiv:hep-ex/0511059].

[10] J. Allison et al., "Geant4 developments and applications,” IEEE Trans. Nucl. Sci. 53 (2006) 270. 